\title{
Perceptual Comparison of Procedural and Data-Driven Eye Motion Jitter
}

\author{
Sophie Jörg \\ Clemson University \\ sjoerg@clemson.edu \\ Krzysztof Krejtz \\ kkrejtz@swps.edu.pl
}

SWPS University of Social Sciences and Humanities

\begin{abstract}
Research has shown that keyframed eye motions are perceived as more realistic when some noise is added to eyeball motions and to pupil size changes. We investigate whether this noise, in contrast to being motion captured, can be synthesized with standard techniques, e.g., procedural or data-driven approaches. In a twoalternative forced choice task, we compare eye animations created with four different techniques: motion captured, procedural, datadriven, and keyframed (lacking noise). Our perceptual experiment uses three character models with different levels of realism and two motions. Our results suggest that procedural and data-driven noise can be used to create animations at similar perceived naturalness to our motion captured approach. Participants' eye movements when viewing the animations show that animations without jitter yielded fewer fixations, suggesting ease of dismissal as unnatural.
\end{abstract}

\section{CCS CONCEPTS}

\section{- Computing methodologies $\rightarrow$ Animation; Perception.}

\section{KEYWORDS}

Character animation, Eye motion, Perceptual evaluation

\section{ACM Reference Format:}

Sophie Jörg, Andrew T. Duchowski, Krzysztof Krejtz, and Anna Niedzielska. 2019. Perceptual Comparison of Procedural and Data-Driven Eye Motion Jitter. In ACM Symposium on Applied Perception 2019 (SAP '19), September 19-20, 2019, Barcelona, Spain. ACM, New York, NY, USA, 5 pages. https: //doi.org/10.1145/3343036.3343130

\section{INTRODUCTION AND BACKGROUND}

Eye motions are an important element of character animation [Ruhland et al. 2014]. The uncanniness of virtual characters has been ascribed to low quality or erroneous eye motions [Hodgins et al. 2010]. Many suggested methods tackle the creation of gaze, typically creating fixations and saccades [Lee et al. 2002] often coupled

Permission to make digital or hard copies of all or part of this work for personal or classroom use is granted without fee provided that copies are not made or distributed for profit or commercial advantage and that copies bear this notice and the full citation on the first page. Copyrights for components of this work owned by others than ACM must be honored. Abstracting with credit is permitted. To copy otherwise, or republish, to post on servers or to redistribute to lists, requires prior specific permission and/or a fee. Request permissions from permissions@acm.org.

SAP '19, September 19-20, 2019, Barcelona, Spain

(C) 2019 Association for Computing Machinery.

ACM ISBN 978-1-4503-6890-2/19/09 . \$ \$15.00

https://doi.org/10.1145/3343036.3343130

\author{
Andrew T. Duchowski \\ Clemson University \\ duchowski@clemson.edu
}

\author{
Anna Niedzielska \\ SWPS University of Social Sciences and Humanities \\ aniedzielska@st.swps.edu.pl
}

with head motions [Andrist et al. 2012; Yeo et al. 2012]. Truoiu et al. [2011] investigate the detailed animation of blinks.

However, the investigation of our perception of more detailed movement such as microsaccades, ocular drift, ocular microtremor, and pupil unrest [Martinez-Conde et al. 2004; Stark et al. 1958] on virtual characters has only started recently. We have shown that animations are perceived as more realistic when a modicum of noise or jitter is added to eye fixations and pupil size [Duchowski et al. 2015, 2016; Jörg et al. 2018; Krejtz et al. 2017]. However, previous research has not addressed to what extent the type of noise matters and which is the best method for its creation. From an animator's perspective, there are three general approaches: keyframed, datadriven, and procedural [Parent 2007]. Keyframed approaches give the animator full control but require more time and skill. Datadriven approaches contain noise implicitly [Deng et al. 2005; Ma and Deng 2009], but they may be less realistic if the noise requires synchronization with other motion. Finally, procedural methods have the advantage that parameters can be adjusted to one's preferences. In this paper we evaluate if jitter generated procedurally or with a data-driven approach achieves similar perceived naturalness to jitter based on motion-captured data, keeping each animation method as simple as possible. We use motion without jitter as a baseline. Our research question is thus: "Are simple data-driven or procedural techniques sufficient to simulate natural jitter?"

In a perceptual experiment, we compare these four animations to provide animators with options for simulating jitter. Our stimuli and computation of saccades is based on Jörg et al.'s work [2018], who determined the amplitude of eyeball and pupil size jitter that was perceived as most natural for three characters and two eye motions. We find that any one of our motion-captured, procedural, or datadriven approaches to synthesize jitter is perceived to be equally natural, and is preferred over animation lacking jitter altogether. This result holds true for our three virtual characters with different levels of realism.

\section{METHOD}

We designed a $4 \times 3 \times 2$ experiment with a two-alternative forced choice task. Our three within-subject variables are Animation: motion captured (MC), procedural (PR), data-driven (DD), and keyframed (KF; no jitter); Character: highly realistic human $(\mathrm{H})$, less realistic and more cartoony human (C), and anthropomorphic robot (R), and Motion: looking at nine distinct points and reading.

We hypothesized that the three techniques that produce jitter, namely motion-captured, procedural, and data-driven, would be 
similar in their perceived naturalness, while the keyframed animation would be perceived as the least natural-looking animation.

\subsection{Creating the Stimuli}

We use four animation techniques: motion-captured, procedural, data-driven, and keyframed (lacking jitter). Each is applied to both eye motion and pupil size at the same time. We start with the captured motions and characters from Jörg et al. [2018]: two motions each applied to three characters. Both eye motions were captured with an eye tracker. The first is similar to a calibration sequence: the performer looked at nine points on a grid on a whiteboard in sequence, fixating each point for two seconds. The second is a reading motion, where text was read on a whiteboard. The motions are 20 and 12 seconds long, respectively. The nine-point motion is characterized by longer fixations and larger saccades compared to the reading motion with shorter fixations and smaller saccades. The virtual characters are a realistic humanlike character, a more cartoony human character, and an anthropomorphic robot that have been created or adjusted and rigged for detailed eye animation.

The motion-captured animation (MC) uses the original captured data and scales down the noise of the eyeball motion within each fixation and of the pupil for the whole take, using scaling factors perceived as most natural, as reported by Jörg et al. [2018].

In the keyframed condition (KF) we set the scaling factor to 0.0 , thereby eliminating all eye motion and pupil size jitter. The general eye motions of the saccades and their timing were retained, simulating an animation that would be created with simple keyframing.

To create jitter for our data-driven condition (DD), we selected pieces of motion from a recorded motion with long fixations that was not used in any of our experiments. We extracted the eye motion jitter from an arbitrary fixation by subtracting the average position of the eye from each gaze point value, scaled it appropriately, and looped it. The extracted data was 100 frames long (at 30 fps). Similarly, we selected an inconspicuous data clip for the pupil size jitter. The resulting data-driven jitter was looped and added to the keyframed condition for the duration of each motion.

Finally, we created jitter for the eyeballs and the pupil size procedurally by applying pink noise to the keyframed animation, following Duchowski et al. [2015], with $\alpha=1.6$ for the eyeballs and $\alpha=0.6$ for the pupils. The noise was created with Matlab's colorednoise generation function and the average jitter amplitudes were adjusted to match the results in Tables 1 and 2 of Jörg et al. [2018].

\subsection{Procedure}

Our experiment is designed as a two-alternative forced choice task, showing two animations at the same time as that design allows for a more detailed comparison than showing videos one by one. Each animation is compared to all others with the character and the eye motion held constant. Videos are presented one above the other (see Figure 1, Left) with randomized but balanced selection (i.e., each video was shown at each position equally as often). This led to 6 (animations) $\times 3$ (characters) $\times 2$ (motions) $=72$ comparisons.

Participants were given information about the experiment, filled out a consent form, answered demographic questions, and the eye tracker (Gazepoint GP3, accurate to about $1^{\circ}$ visual angle) was calibrated ( 5 points). The forced-choice task then started and the
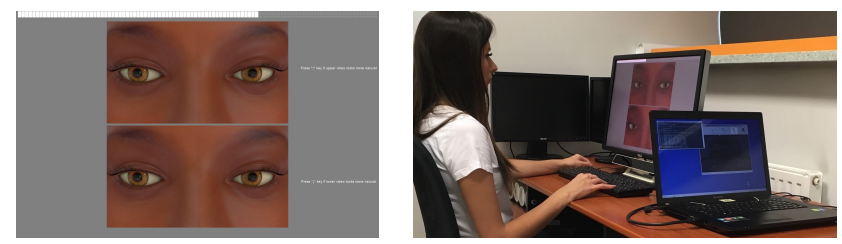

Figure 1: Left: Screenshot from our experiment: the videos, in this case our cartoony human, are shown in synchrony one on atop the other. Instructions are displayed on the right. A progress bar at top indicates that about two thirds of the experiment is over. Right: Experimental setting (SWPS University). The participant is evaluating two videos with gaze recorded on a separate laptop.

participant was asked to select the animation that "looks most natural" each time. The two videos were played in synchrony. The vertical video arrangement facilitated eye motion comparison as compared to side-by-side arrangement, which we used in early tests of this experiment and resulted in participants comparing the two eyes in the center. The animations were looped and had to be watched for a minimum of 10 seconds each. A progress bar informed the participant about the remaining experiment time. Participants were debriefed and asked for comments at the end.

\subsection{Participants}

The experiment was conducted at two institutions in parallel: Clemson University and the SWPS University of Social Sciences \& Humanities, with consistent procedures and apparatus at both locations. At Clemson University, 21 participants (9f, 12m) aged 18-35 $(\mathrm{M}=24.1, \mathrm{SD}=3.9)$ took part in our study in exchange for a voucher equivalent to a coffee, sandwich or other small reward. The data of one participant was removed from further analyses due to technical problems. Thus the final sample consisted of 20 participants (9f, $11 \mathrm{~m})$ aged between 18 and 35 years old $(\mathrm{M}=24.2$, $\mathrm{SD}=3.97)$. Eighteen volunteers $(13 \mathrm{f}, 5 \mathrm{~m})$ between 20 and 42 years of age $(M=25.3 \mathrm{SD}=7.1)$ participated in the experiment at our second location in exchange for academic credit points.

Both experiments used a GP3 eye-tracker at $60 \mathrm{~Hz}$, and PsychoPy software to control the experimental procedure. Screen resolution was set to $1920 \times 1200$ with participants seated at a distance of about $57 \mathrm{~cm}$ (see Figure 1, Right). Most participants finished the study in 20-30 minutes.

\section{RESULTS}

Analyses of behavioral data (participants' selections) are based on goodness-of-fit Chi-squared $\left(\chi^{2}\right)$ tests, due to the binomial nature of the data. If the $\chi^{2}$ test reveals a significant effect, it is followed by pairwise comparisons to determine which differences are significant. Finally, we use exact binomial tests to compare each stimuli proportion of choices to random selection 0.5 [Conover 1971].

To analyze the behavioral data we removed outliers based on the time to make the selection. Outliers were identified with the standard $1.58 \times$ IQR formula (IQR: interquartile range). 239 points $(8.74 \%)$ were identified as outliers with mean $23.96 \mathrm{sec} .(\mathrm{SD}=12.13)$. 

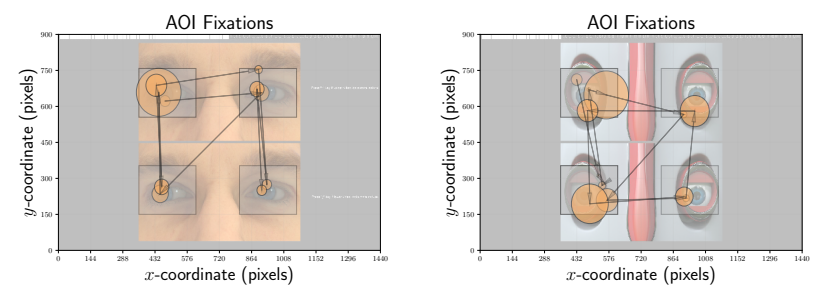

Figure 2: Exemplary eye movement data scanpaths for realistic human (left) and anthropomorphic robot model (right).

Reasons for such long decision times may be the participant's loss of focus. We therefore expect random distribution of participants' choices in these trials. We confirmed this prediction with a Chisquared test. There were no significant differences between choices of animation type, $\chi^{2}(3)=3.16, p \approx 0.37$.

In order to analyze the distribution of visual attention between the different eye movement animations, we first drew Areas of Interest (AOIs) around the eyes for each animation, see Figure 2. Eye movement data for AOIs around both left and right eyes on the same character were grouped. Because two animations were presented simultaneously on the screen, we removed from the data the first and last fixations on each animation, as these fixations were thought to be related to attention shifts toward a new stimulus. We also removed all fixations with duration less than $80 \mathrm{~ms}$ or greater than $1000 \mathrm{~ms}$, which is standard procedure for eye tracking data to remove artifacts related to, for example, blinks.

\subsection{Analysis of Participants' Selections}

Naturalness of Animation. To evaluate how often each animation technique was selected as most natural, we identified the chosen animation technique in each forced-choice task. The Chi-squared goodness-of-fit test revealed that the proportion of selection differed in at least one of the stimuli, $\chi^{2}(3)=23.29, p<0.001$. The following pairwise comparisons showed that the keyframed animation was selected as natural (42\%) significantly less often $(p<0.001)$ than the data-driven (54\%), the procedural (53\%), or the motioncaptured (52\%) animations, see Figure 3, Left, thus confirming our hypothesis that the keyframed animation would be selected least often as the more natural-looking animation. We did not see significant differences in frequency of selections between data-driven, procedural and motion-captured animations $(p>0.1)$.

Exact binomial tests showed that the proportion of selection of DD is significantly greater than $0.5(p<0.001)$, and significantly lower than $0.5(p<0.001)$ for the KF. The proportion of selection of PR was higher than 0.5 but only marginally significant $(p=0.079)$.

Selections for Each Character Model. Next, we compared selection between motion techniques for each character model.

For the robot model and the cartoony model, the goodness-offit Chi-squared tests revealed significant differences of selection between animation techniques with $\chi^{2}(3)=12.86, p<0.01$ and $\chi^{2}(3)=8.90, p<0.05$, respectively. The following multiple comparisons showed that for both characters the keyframed animation was significantly $(p<0.05)$ less often evaluated as more natural
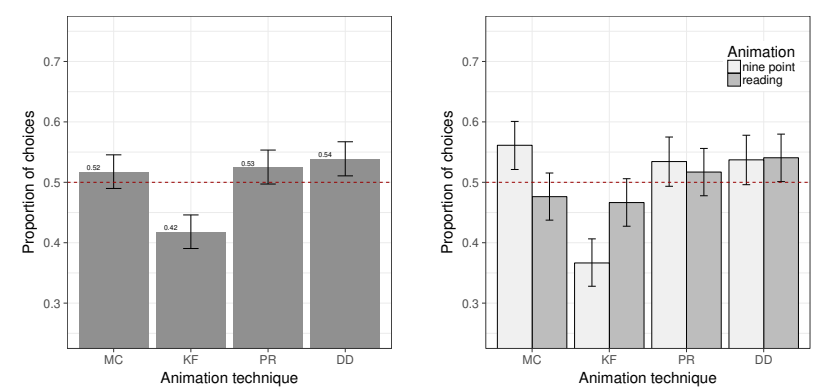

Figure 3: Choice data analyses results for which animation is perceived as more natural with MC: motion capture, KF: keyframed, DD: data-driven, and PR: procedural. Left: Animation techniques overall; Right: Animation techniques per motion (nine point vs. reading). Whiskers represent $95 \%$ intervals from exact binomial tests.

in comparison to all other animation techniques except that the difference between the conditions $\mathrm{MC}$ and $\mathrm{KF}$ was only marginally significant for the cartoony character with $p=0.06$.

Exact binomial tests showed that KF was selected significantly less often $(p<0.001)$ than random for both models whereas DD was selected significantly more often than random with $p<0.05$.

For the realistic human model the Chi-squared did not reach statistical significance, $\chi^{2}(3)=5.27, p>0.1$. However, exact binomial tests showed that the proportion of selection of the keyframed animation was significantly lower $(p<0.02)$ than random. The proportion of selection of the motion-captured animation differed from random but only marginally $(p=0.077)$.

Selections for Each Motion. We then performed two separate Chisquared tests, one for each motion.

For the nine point eye motion, the Chi-squared test was statistically significant, $\chi^{2}(3)=30.58, p<0.001$. Again, the keyframed animation was selected significantly less often than the other animation techniques $(p<0.001)$. Exact binomial tests revealed that KF was selected significantly less often than random and MC significantly more often than random ( $p<0.001$ for both). Selection of DD was only marginally ( $p=0.077$ ) different from random.

For the reading eye motion, the Chi-squared test was not statistically significant, $\chi^{2}(3)=4.32, p>0.1$. KF and DD were selected less often and more often than random, respectively, at marginally significant levels ( $p=0.097$ and $p=0.051$ ), see Figure 3, Right.

Decision Time. To find out if the displayed motion or character model influenced the time to make a decision, we conducted a two-way ANOVA with decision time as the dependent variable and motion and character as within-subjects fixed factors. Overall, the average time taken to choose the animation that looked most natural was 2.95 seconds ( $\mathrm{SD}=3.07$ ), not including the 10 seconds participants had to wait before being able to make a selection.

Results showed a significant main effect of motion $F(1,37)=$ 27.63, $p<0.001, \eta^{2}=0.05$. Decision time was on average significantly longer for the nine point animation $(M=3.27 \mathrm{sec} ., \mathrm{SD}=$ $3.85)$ than for the reading animation $(M=2.65 \mathrm{sec} ., \mathrm{SD}=3.81)$. 

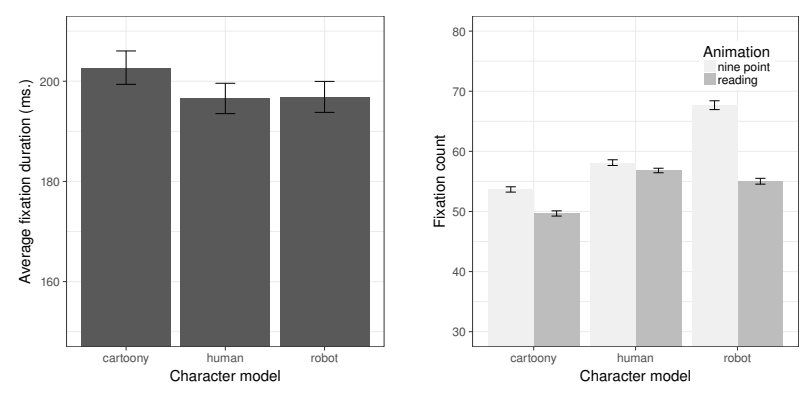

Figure 4: Eye movement data analyses. Left: Comparison of average fixation duration on the different characters; Right: Comparison of fixation count between character split by motion. Whiskers represent $95 \%$ confidence intervals.

\subsection{Analysis of Participants' Eye Movements}

Eye tracking data analyses used three-way within-subjects analyses of variance (ANOVA) with a $3 \times 2 \times 4$ design with character, motion and animation as within-subjects fixed factors. The ANOVAs were followed by pairwise comparisons with Tukey HSD correction, for any statistically significant effects detected. Dependent measures included fixation duration (a measure of depth of visual processing) and average fixation count (a measure of attention distribution).

Fixation duration. ANOVA revealed marginally significant main effects of motion $\left(F(1,31)=3.83, p=0.06, \eta^{2}=0.002\right)$ and character $\left(F(2,62)=3.14, p=0.05, \eta^{2}=0.003\right)$. Average fixation duration was significantly longer for reading motions $(M=200.7$ ms., $S E=1.56)$ than for the nine point motions $(M=196.4 \mathrm{~ms}$., $\mathrm{SE}=1.46)$. Pairwise comparisons showed that fixation durations were significantly longer $(p<0.05)$ on the cartoony character than on the robot and on the realistic human, see Figure 4, Left.

Fixation count. ANOVA revealed significant main effects of motion and character $\left(F(1,31)=8.73, p<0.01, \eta^{2}=0.006 ; F(2,62)=\right.$ $\left.3.26, p<0.05, \eta^{2}=0.008\right)$. The nine point motions gathered significantly more fixations $(\mathrm{M}=60.1 \mathrm{~ms} ., \mathrm{SE}=0.23)$ compared to the reading motions $(M=54.0, \mathrm{SE}=0.17)$. Pairwise comparisons showed that the cartoony character drew significantly fewer $(p<0.05)$ fixations $(\mathrm{M}=51.7, \mathrm{SE}=0.18)$ than the realistic human $(\mathrm{M}=57.4, \mathrm{SE}=0.17)$ and the $\operatorname{robot}(\mathrm{M}=61.9, \mathrm{SE}=0.27)$.

Analysis also showed a significant interaction effect of character and motion $\left(F(2,62)=3.45, p<0.05, \eta^{2}=0.005\right)$, see Figure 4, Right. Pairwise comparisons revealed a significant difference $(p<$ 0.05 ) in fixation count between the nine point and reading motions for the robot character as well as for the cartoony character, whereas there was no such difference for the human character with $p>0.1$.

Moreover a three-way ANOVA showed a marginally significant interaction effect of character and animation $(F(4.40,136.38)=2.06$, $\left.p=0.08, \eta^{2}=0.004\right)$. Pairwise comparisons showed that for the robot, the fixation count when watching DD was higher than for PR at statistical tendency $(p=0.057)$. For the cartoony character, DD had significantly less fixations than MC $(p<0.02)$. The difference between the DD and PR reached statistical tendency $(p=0.061)$ For the human character, KF was fixated significantly less $(p<0.01)$ often than MC.

\section{DISCUSSION AND CONCLUSION}

Our most important new finding over previous work is that any one of our motion-captured, procedural, or data-driven approaches to synthesize jitter is perceived to be equally natural, and is preferred over animation lacking jitter altogether. Our take home message is therefore that all three animation techniques - motion captured, procedural, and data-driven - can be used when creating jitter to animate compelling eye motions.

For all character models and eye motions used in the study, the keyframed condition was perceived by participants as the least natural. This finding corroborates earlier work [Krejtz et al. 2017] who found that eye motions with some types of procedural jitter and some attenuated motion captured eye motions were preferred over eye movements without any jitter. Interestingly, in our study, keyframed eye animations drew fewer fixations compared to the other animations, suggesting ease of decision making when rejecting them as unnatural. The remaining animations received nearly the same number of fixations during decision making.

Adding jitter to eye movements may be particularly important for animations where the character is attempting to hold gaze steady, i.e., when fixating, such as in the nine point motion. Analyzing decision times showed that the nine point motion animations took significantly more time to evaluate. Nine point has longer segments of motion in which the eyes do not move much. During those motion segments the jitter might be more salient, encouraging participants to spend more time looking at those details and making a decision. This may explain why participants were able to distinguish between the different eye motions with greater disparity when viewing the nine point than when viewing the reading animations. Furthermore, the fixations were shorter and there were more of them for the nine point motions, suggesting that the decisions were easier for the participants than for the reading motions.

Our results are very similar for our three characters with different levels of realism, but some findings such as the selection of the more natural animation and the differences between the reading and nine point motions concerning the fixation count are less pronounced for the realistic human, which could indicate that adding jitter is even more important for less realistic characters. Analyses of viewers' eye gaze showed furthermore that the cartoony character's eye animations required longer fixation durations compared to the other animated characters. Prolonged average fixation duration is usually associated with more extensive cognitive processing of visual stimuli which in the present study may suggest difficulty in decision making.

Finally, our results indicate that our approach can be used to build a tool for animators to enhance keyframed eye animations with jitter using a controllable parameter to adjust the jitter magnitude. Based on our results, such a tool can use a procedural or data-driven approach without noticeable perceptual difference in naturalness from scaled motion capture data. Future work could investigate if adjusting such a parameter could be used to convey the emotions or state of mind of a virtual character.

\section{ACKNOWLEDGMENTS}

This material is based upon work supported by the National Science Foundation under Grant Number IIS-1423189. 


\section{REFERENCES}

Sean Andrist, Tomislav Pejsa, Bilge Mutlu, and Michael Gleicher. 2012. A Head-eye Coordination Model for Animating Gaze Shifts of Virtual Characters. In Proceedings of the 4th Workshop on Eye Gaze in Intelligent Human Machine Interaction (Gaze-In '12). ACM, New York, NY, USA, Article 4, 6 pages. https://doi.org/10.1145/2401836. 2401840

William J. Conover. 1971. Practical Nonparametric Statistics. John Wiley \& Sons, New York

Zhigang Deng, J. P. Lewis, and Ulrich Neumann. 2005. Automated eye motion using texture synthesis. IEEE Computer Graphics Applications 25, 2 (March 2005), 24-30. https://doi.org/10.1109/MCG.2005.35

Andrew Duchowski, Sophie Jörg, Aubrey Lawson, Takumi Bolte, Lech Świrski, and Krzysztof Krejtz. 2015. Eye movement synthesis with $1 / f$ pink noise. In Proceedings of the 8th ACM SIGGRAPH Conference on Motion in Games (MIG '15). 47-56. https: //doi.org/10.1145/2822013.2822014

Andrew T. Duchowski, Sophie Jörg, Tyler N. Allen, Ioannis Giannopoulos, and Krzysztof Krejtz. 2016. Eye movement synthesis. In Proceedings of the Ninth Biennial ACM Symposium on Eye Tracking Research \& Applications (ETRA '16). 147-154. https://doi.org/10.1145/2857491.2857528

Jessica Hodgins, Sophie Jörg, Carol O’Sullivan, Sang Il Park, and Moshe Mahler. 2010. The Saliency of Anomalies in Animated Human Characters. ACM Transactions on Applied Perception (TAP) 7, 4, Article 22 (July 2010), 22:1-22:14 pages. https: //doi.org/10.1145/1823738.1823740

Sophie Jörg, Andrew Duchowski, Krzysztof Krejtz, and Anna Niedzielska. 2018. Perceptual Adjustment of Eyeball Rotation and Pupil Size Jitter for Virtual Characters. ACM Trans. Appl. Percept. 15, 4, Article 24 (Oct. 2018), 13 pages. https: //doi.org/10.1145/3238302
Krzysztof Krejtz, Andrew Duchowski, Heng Zhou, Sophie Jörg, and Anna Niedzielska. 2017. Perceptual evaluation of synthetic gaze jitter. Computer Animation and Virtual Worlds (2017), e1745. https://doi.org/10.1002/cav.1745

Sooha Park Lee, Jeremy B. Badler, and Norman I. Badler. 2002. Eyes alive. ACM Transactions on Graphics 21, 3 (July 2002), 637-644. https://doi.org/10.1145/566654. 566629

Xiaohan Ma and Zhigang Deng. 2009. Natural eye motion synthesis by modeling gaze-head coupling. In IEEE Virtual Reality. Lafayette, LA, 143-150. https://doi. org/10.1109/VR.2009.4811014

Susana Martinez-Conde, Stephen L. Macknik, and David H. Hubel. 2004. The role of fixational eye movements in visual perception. Nature Reviews Neuroscience 5, 3 (March 2004), 229-240. https://doi.org/10.1038/nrn1348

Rick Parent. 2007. Computer Animation: Algorithms and Techniques (3 ed.). Elsevier, Waltham, MA, USA.

Kerstin Ruhland, S. Andrist, J. B. Badler, Christopher E. Peters, Norman I. Badler, Michael Gleicher, Bilge Mutlu, and Rachel McDonnell. 2014. Look me in the eyes: A survey of eye and gaze animation for virtual agents and artificial systems. In Eurographics 2014 - State of the Art Reports, Sylvain Lefebvre and Michela Spagnuolo (Eds.). https://doi.org/10.2312/egst.20141036

Lawrence Stark, Fergus W. Campbell, and John Atwood. 1958. Pupil unrest: An example of noise in a biological servomechanism. Nature 182, 4639 (1958), 857-858.

Laura C. Trutoiu, Elizabeth J. Carter, Iain Matthews, and Jessica K. Hodgins. 2011. Modeling and animating eye blinks. ACM Transactions on Applied Perception (TAP) 2, 3, Article 17 (May 2011), 17 pages. https://doi.org/10.1145/2010325.2010327

Sang Hoon Yeo, Martin Lesmana, Debanga R. Neog, and Dinesh K. Pai. 2012. Eyecatch: Simulating visuomotor coordination for object interception. ACM Transactions on Graphics 31, 4, Article 42 (July 2012), 10 pages. https://doi.org/10.1145/2185520. 2185538 